LESSON OF THE MONTH

\title{
The importance of suspecting superficial siderosis of the central nervous system in clinical practice
}

\author{
A Messori, P Di Bella, N Herber, F Logullo, M Ruggiero, U Salvolini
}

J Neurol Neurosurg Psychiatry 2004;75:188-190. doi: 10.1136/jnnp.2003.023648

Once the central nervous system surface is greatly encrusted with haemosiderin, even removing the source of bleeding will have little effect on the progression of clinical deterioration. Superficial siderosis of the central nervous system is rare and insidious, but magnetic resonance imaging has turned a previously late, mainly autoptical diagnosis into an easy, specific, in vivo, and possibly early one. Avoiding long diagnostic delay will be very important in those cases susceptible of causal treatment.

l: t has become possible to diagnose superficial siderosis (SS) of the central nervous system (CNS), a rare and insidious cause of progressive neurological deterioration, with magnetic resonance imaging (MRI), thanks to the pathognomonic finding of diffuse hypo-intensity at the surface of cerebellum, brain stem, inferior part of cerebral hemispheres, and spinal cord on spin echo T2 weighted and gradient echo $\mathrm{T} 2 *$ weighted images. Both sequences, the latter with higher sensitivity, are suited for detecting haemosiderin encrusting the leptomeninges and surface of brain cortex. ${ }^{1-4}$ Identification of the source of chronic bleeding into the subarachnoid spaces, which is obtained in about half of cases, may lead to removal preventing further accumulation of blood derivatives around nervous structures. However, because of its rarity and the slow progression of symptoms (typically deafness and ataxia), SS is still likely to be diagnosed late. If this is the case, even successful causal treatment will have little effect on the progression of clinical deterioration. A patient with SS of the CNS due to myxopapillary ependymomas of the cauda equina is reported herein, because his case yields information about the typical history, presentation, and MRI findings of this disease, and also calls attention to possible related additional problems.

\section{CASE REPORT}

A 65 year old man was admitted for evaluation of his bilateral deafness and difficulty walking, requiring support, which had progressed over a 20 year period. Five years earlier, computerised tomography head scanning had shown no abnormalities except for atrophic changes, which had been considered consistent with age. His history was otherwise unremarkable. Neurological examination revealed severe neurosensory bilateral hypoacousia, mixed cerebellar and sensory ataxia with severe gait impairment, horizontal nystagmus, vertigo, bilateral dysmetry, paraparesis with hyperreflexia and extensor plantar responses, lower limb numbness, loss of vibration sense and altered joint position sense bilaterally, and wasting of distal leg muscles. Electromyography-electroneurography showed bilateral L4-L5-S1 radiculopathy with active denervation in leg and foot muscles. Brain magnetic resonance imaging (MRI) performed with 1.0-T magnet (GE, Milwaukee, WI, USA) showed especially cerebellar marked atrophy; fast spin echo T2 weighted and gradient echo T2* weighted images showed a rim of hypointensity all around the cerebellum, brainstem, and interhemispheric and sylvian fissures (fig lA-D). In the absence of any detectable intracranial abnormality as source of bleeding into the subarachnoid space, MRI of the spine was carried out. It showed diffuse hypointensity at the surface of the whole cord, and intradural extramedullary expanding lesions at L2 to L3 and L4 levels, respectively. These appeared ovoid in shape and adherent to the nerve roots, gave inhomogeneous signal due to intrinsic isohyperintensity and concomitant hypointensities consistent with haemosiderin, and showed marked enhancement after intravenous gadolinium DTPA (fig $1 E-G$ ). They were surgically removed, and pathology yielded myxopapillary ependymomas. Recovery was complicated by bilateral subdural haematomas, which required surgical evacuation and began resolving only after a two month inpatient treatment.

\section{DISCUSSION}

Although superficial siderosis of the central nervous system was first described as far back as 1908 and its pathogenetic mechanism and clinical manifestations were a matter of pathological and neurological papers in the 1960s, ${ }^{356}$ it is likely to be not yet familiar to clinicians, because it is rare and generally not described in neurological texts. About one hundred cases have been reported to date, initially following rare autoptical or surgical observation, and far more frequently in the late 1980s and 1990s, thanks to the "revolution" in diagnosis provided by MRI. ${ }^{35-8}$ It is caused by deposition of blood by products in the leptomeninges, subpial tissue of the brain and spinal cord, and cranial nerves as a result of haemorrhage in the subarachnoid space. ${ }^{9}$ It may be a late complication of hemispherectomy, previous clinically evident haemorrhages, and surgery, but more often it is caused by repeated, even small subclinical bleeds. The source of bleeding is found in about half of cases; however, diffusion and greater use of modern imaging tools will probably allow for more frequent detection of the underlying abnormalities, and they are making it possible to diagnose and treat these early, thus also preventing the development of clinically significant secondary SS. ${ }^{34}$ Nevertheless, the origin of SS will probably still remain undetected in some cases.. ${ }^{410}$ Highly vascular spinal tumours, most frequently ependymomas, and CNS vascular

Abbreviations: CNS, central nervous system; MRI, magnetic resonance imaging; SS, superficial siderosis 

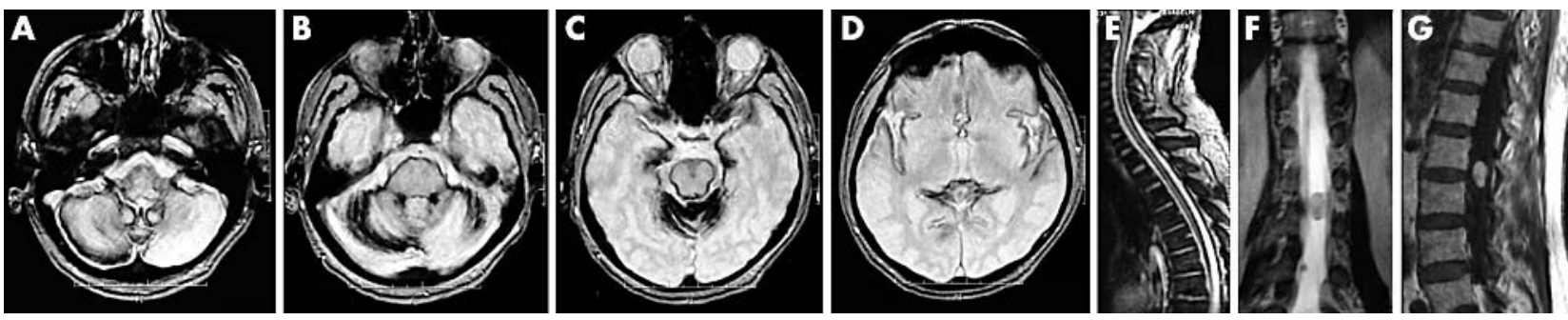

Figure 1 (A-D) Gradient echo T2*-weighted axial MRI of the brain shows a rim of hypointensity (consistent with the presence of haemosiderin deposits in the leptomeninges and subpial tissue) on the surface of cerebellum, brain stem, and inferior part of cerebral hemispheres (sylvian and interhemispheric fissures, and basal cisterns). Please note the particular involvement of superior cerebellar folia (B, C) and the hypointensity on eighth (A) and, less conspicuously, fifth (B) cranial nerves bilaterally. (E-G) MRI of the spine. (E) T2 weighted sagittal image shows a hypointense rim surrounding the spinal cord. (F) T2 weighted coronal image at lumbar level demonstrates intradural extramedullary expanding lesions at L2-L3 and L4 levels, respectively, giving inhomogeneous signal due to intrinsic iso-hyperintensity and concomitant hypointensities consistent with haemosiderin. (G) On T1 weighted midsagittal image after intravenous gadolinium DTPA, the expanding lesion at L2-L3 level appears markedly enhanced, as did the minor lateral lesion at L4 level. This feature, as well as the presence of intrinsic haemosiderin, is consistent with the high vascularity of myxopapillary ependymomas, a subtype of glioma occurring almost exclusively in the filum terminale and conus medullaris, and accounting for nearly $90 \%$ of primary tumours in the cauda equina region.

abnormalities are the sources of chronic bleeding most frequently found in these patients.

Sensorineural hearing loss and cerebellar ataxia are the typical clinically relevant picture; myelopathy and dementia are less common, while other probably frequent symptoms (for example, anosmia) are likely to be underdiagnosed. Previous papers have addressed their pathogenesis and the selective involvement of cerebellum, brain stem, sylvian and temporal brain cortex, and cranial nerves, in terms of either intrinsic vulnerability or anatomical position. ${ }^{369}$ The progression of symptoms is generally very slow and several years, even decades, may pass before medical attention is sought. Owing to this factor and to their lack of specificity, SS of CNS was probably underdiagnosed in the past, when mainly pathologists (and, rarely, surgeons) had some awareness of it. Since the advent of MRI, it has been more frequently, and even in subclinical cases, described by neuroradiologists, thanks to the evidence and specificity of its findings. However, particularly when not so conspicuous, these are likely to be somewhat overlooked unless they are correlated with clinical history and observation. On the other hand, the diagnosis will be missed unless patients with these non-specific symptoms undergo MRI study, because computerised tomography is not suited for detecting haemosiderin.

The patient reported here is a representative example of the typical clinical history of SS of the CNS, with SS secondary to bleeding from myxopapillary ependymomas, caudal highly vascular glial tumours frequently associated with intra- and/ or extratumoural bleeding. It shows how worrying a delayed diagnosis of secondary SS may be in cases like this one, where a slow growing, bleeding lesion with quite favourable prognosis following complete surgical resection could have been removed earlier, with earlier and more specific diagnostic investigation. Late diagnosis and treatment are very likely to have little impact on the patient's condition. Tentative treatment of SS per se with iron chelating agents $^{369}$ has not proved useful, and removal of the causal illness will probably only slow down the progression of the pathological process and clinical deterioration.

Our patient's myxopapillary ependymomas were otherwise asymptomatic, as these tumours often are, so that they are often diagnosed late. Whenever SS is diagnosed with brain MRI in a patient without any detectable intracranial source of bleeding or history consistent with it, imaging the spine is mandatory, and it should also be recommended with subarachnoid haemorrhage of unknown origin; early diagnosis of a silent spinal tumour may make surgery more successful.

We would also like to draw attention to this patient's postsurgical complication-that is, subdural haematomas and an abnormally long time to their resolution after surgical evacuation: although his cerebral hemispheres, consistent with the literature findings, were relatively less conspicuously involved by SS as shown by MRI, some diffuse abnormal "rigidity" of the brain covers and surface might have played a role. On this basis, SS of the CNS might be considered as a risk factor for subdural haematoma.

To conclude, it is important to suspect superficial siderosis of the central nervous system in clinical practice in order to avoid delay in diagnosis and treatment, when possible. Even slight MRI features consistent with an initial degree of SS should not be underestimated, in order to avoid severe irreversible damage to CNS.

\section{Authors' affiliations}

A Messori, N Herber, M Ruggiero, U Salvolini, Department of Neuroradiology, Umberto I Hospital and University of Ancona, Torrette, Ancona, Italy

P Di Bella, F Logullo, Department of Neurology, Umberto I Hospital and University of Ancona, Torrette, Ancona, Italy

Competing interests: none declared

Correspondence to: Prof U Salvolini, Department of Neuroradiology, Ospedale Umberto I e Università degli Studi di Ancona, via Conca, Torrette, 60020 Ancona, Italy; u.salvolini@popcsi.unian.it

Received 16 July 2003

Accepted 16 July 2003

\section{REFERENCES}

1 Gomori JM, Grossman RI, Bilaniuk LT, et al. High-field MR imaging of superficial siderosis of the central nervous system. J Comput Assist Tomogr 1985:9:972-5

2 Zimmerman RA, Hesselink JR, Bilaniuk LT, et al. Bilateral pial siderosis and hearing loss: syndrome with negative CT and positive high field MRI findings. Radiology 1988;169(P):312.

3 Bracchi M, Savoiardo M, Triulzi F, et al. Superficial siderosis of the CNS: MR diagnosis and clinical findings. AJNR Am J Neuroradiol 1993;14:227-36.

4 Offenbacher H, Fazekas F, Schmidt R, et al. Superficial siderosis of the central nervous system. MRI findings and clinical significance. Neuroradiology 1996;38:551-6.

5 Willeit J, Aichner F, Felber S, et al. Superficial siderosis of the central nervous system: report of three cases and review of the literature. J Neurol Sci 1992;111:20-5.

6 Fearnley JM, Stevens JM, Rudge P. Superficial siderosis of the central nervous system. Brain 1995;118:1051-66. 
7 Lemmerling M, De Praeter G, Mollet P, et al. Secondary superficial siderosis of the central nervous system in a patient presenting with sensorineural hearing loss. Neuroradiology 1998;40:312-4.

8 Hsu WC, Loevner LA, Forman MS, et al. Superficial siderosis of the CNS associated with multiple cavernous malformations. AJNR Am J Neuroradiol 1999:20:1245-8.
9 Koeppen AH Dentinger MP. Brain haemosiderin and superficial siderosis of the central nervous system. I Neuropathol Exp Neurol 1988;47:249-70.

10 Bourgouin PM, Tampieri D, Melancon D, et al. Superficial siderosis of the brain following unexplained subarachnoid hemorrhage: MRI diagnosis and clinical significance. Neuroradiology 1992;34:407-10.

\section{Clinical Evidence-Call for contributors}

Clinical Evidence is a regularly updated evidence based journal available worldwide both as a paper version and on the internet. Clinical Evidence needs to recruit a number of new contributors. Contributors are health care professionals or epidemiologists with experience in evidence based medicine and the ability to write in a concise and structured way.

\section{Currently, we are interested in finding contributors with an interest in} the following clinical areas:

Altitude sickness; Autism; Basal cell carcinoma; Breast feeding; Carbon monoxide poisoning; Cervical cancer; Cystic fibrosis; Ectopic pregnancy; Grief/bereavement; Halitosis; Hodgkins disease; Infectious mononucleosis (glandular fever); Kidney stones; Malignant melanoma (metastatic); Mesothelioma; Myeloma; Ovarian cyst; Pancreatitis (acute); Pancreatitis (chronic); Polymyalgia rheumatica; Post-partum haemorrhage; Pulmonary embolism; Recurrent miscarriage; Repetitive strain injury; Scoliosis; Seasonal affective disorder; Squint; Systemic lupus erythematosus; Testicular cancer; Varicocele; Viral meningitis; Vitiligo However, we are always looking for others, so do not let this list discourage you.

Being a contributor involves:

- Appraising the results of literature searches (performed by our Information Specialists) to identify high quality evidence for inclusion in the journal.

- Writing to a highly structured template (about 2000-3000 words), using evidence from selected studies, within 6-8 weeks of receiving the literature search results.

- Working with Clinical Evidence Editors to ensure that the text meets rigorous epidemiological and style standards.

- Updating the text every eight months to incorporate new evidence.

- Expanding the topic to include new questions once every 12-18 months.

If you would like to become a contributor for Clinical Evidence or require more information about what this involves please send your contact details and a copy of your CV, clearly stating the clinical area you are interested in, to Claire Folkes (cfolkes@bmigroup.com).

\section{Call for peer reviewers}

Clinical Evidence also needs to recruit a number of new peer reviewers specifically with an interest in the clinical areas stated above, and also others related to general practice. Peer reviewers are health care professionals or epidemiologists with experience in evidence based medicine. As a peer reviewer you would be asked for your views on the clinical relevance, validity, and accessibility of specific topics within the journal, and their usefulness to the intended audience (international generalists and health care professionals, possibly with limited statistical knowledge). Topics are usually 2000-3000 words in length and we would ask you to review between 2-5 topics per year. The peer review process takes place throughout the year, and our turnaround time for each review is ideally 10-14 days.

If you are interested in becoming a peer reviewer for Clinical Evidence, please complete the peer review questionnaire at www.clinicalevidence.com or contact Claire Folkes(cfolkes@bmigroup.com). 